\title{
Assembly Features Utilization to Support Production System Adaptation
}

\author{
Baha Hasan $^{1, *}$, Mauro Onori ${ }^{2}$, and Jan Wikander ${ }^{1}$ \\ ${ }^{1}$ Department of Machine Design, The Royal Institute of Technology (KTH), \\ Stockholm, Sweden \\ ${ }^{2}$ Department of Production Engineering, The Royal Institute of Technology (KTH), \\ Stockholm, Sweden
}

\begin{abstract}
The purpose of this paper is to introduce a proposed methodology to extend the evolvable assembly system (EAS) paradigm for product design by utilizing assembly features in a product. In this paper, assembly features are used to bridge the gap between product design and assembly process by matching features of a part in an assembly to operations of a process in the EAS ontology. This can be achieved by defining and extracting a new set of assembly features called process features, which are features significant to specific and well- defined assembly operations. The extracted assembly features are represented in a proposed model based on product topology. A case-study example is conducted to illustrate the new methodology. A process-feature ontology is proposed as well in order to match the assembly requirements represented by process features with the available processes and skills in the EAS ontology so that adaptation of the production system can be achieved.
\end{abstract}

Keywords: Adaptation, Evolvable, Assembly, Features, EAS, Process, Skill, Ontology.

\section{Introduction}

Modern production system paradigms have to cope with several critical issues arising from the need for mass customization, such as short product life-cycles, an increasing number of product variants and frequently changing customer requirements. To overcome these challenges, production systems have to be more adaptive such that they can rapidly respond to the required changes.

Several approaches [1], [2], [3] have been proposed in order to support adaptivity of production systems. One of these approaches is the Evolvable Assembly System (EAS) approach, which was proposed in 2002 and developed during the EUPASS project. EAS aims to cope with unpredictable and changing production requirements by building evolvable capabilities into the production system. As stated by Onori et al. [4], [5], [6] evolvability is not only the ability of system components to adapt to changing requirements, but also a characteristic, which assists the processes in

\footnotetext{
* Corresponding author.
} 
becoming more self-x, which can stand for self-evolvable, self-reconfigurable, selftuning, self-diagnosing and so on.

The aim of this paper is to introduce a method to utilize assembly features in an assembly to determine the required assembly process and resource capabilities in the evolvable assembly system (EAS) [7], [8]. In this paper an approach is proposed to improve the adaptability of the evolvable systems by extending the EAS paradigm to the design of a product based on its assembly features.

Assembly features are defined as "features with significance for assembly processes", and are divided into mating (connection) features (such as final position, insertion path/point, tolerances), handling features, (characteristics that give the locations on an assembly component such that it can be safely handled by a gripper during assembly) [9], and form features, which are "A set of geometric entities (surfaces, edges, and vertices) together with specifications of the bounding relationship between them and which have engineering/functional implications and/or provide assembly aid, such as a center line of a hole, on an object'" [10], [11]. In other words, form features are geometrical mating entities, which include mating features. Figure 1 illustrates assembly features definition.

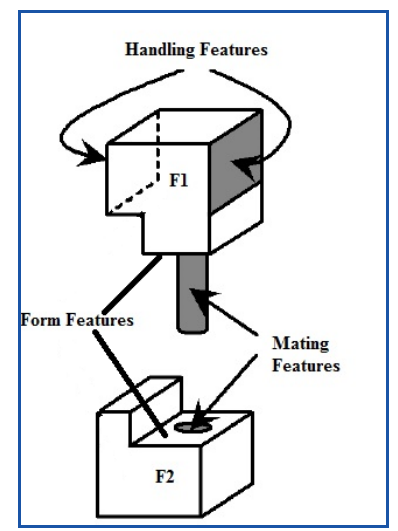

Fig. 1. Assembly features, modified from [12]

In this paper, a methodology is proposed to convert assembly requirements into required assembly processes and capabilities through analysis of assembly features. In this context assembly requirements are those assembly features or characteristics which require a set of moving and joining processes in order to transform a set of components to an assembled or semi- assembled product. An assembly-feature based model is proposed based on definition, representation and extraction of handling, mating and form features. Based on these extracted features, a new set of joining and transporting (moving) features are defined, represented and modeled in an ontology (process-feature ontology) in order to define, in details, the required assembly process and resource capabilities, which will be matched with the available processes and resources in the EAS ontology [7]. The final ultimate aim is to determine and configure the new recourses (modules) in the EAS system, which will be involved to assemble a product. 


\section{Relationship to Collective Awareness Systems}

As EAS is a modular self-reconfigurable system, the proposed methodology tries to create a link between product's assembly features and EAS resource modules represented by their Skills (- Skill is defined as the ability of a resource to perform a process [13]). That is, product and production /assembly systems become linked. In this way product designers become aware of production implications and vice-versa. This enables several stakeholders to share vital information, create awareness and even automate complex development phases (designers, sub-contractors, system integrators, etc.). Figure 2 illustrates this collective awareness link between assembly design, process and EAS.

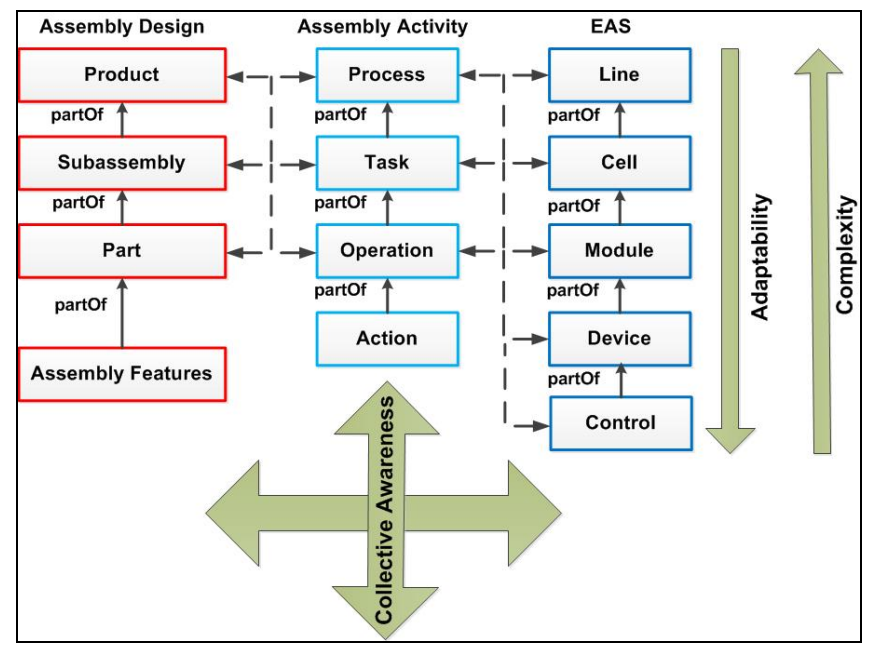

Fig. 2. Connection of design, process and EAS levels, modified from [14]

In Figure 2, the assembly design is composed of subassemblies, being stable assemblies containing two or more parts [14], and parts, which are the elementary components of an assembly [14]. An assembly activity is composed of assembly processes which collections of lower level assembling activities with a purpose of facilitating the assembling of an assembly or subassembly [15], and they are composed of tasks, operations and actions. The EAS is composed of several units; the most fundamental unit is the module unit, which is defined as "a self-composed entity with a given functionality and with well-defined interfaces, via which it interacts with other modules" [4]. In terms of collaborative design, our aim is to facilitate knowledge transfer between part, operation and modules by utilizing assembly features to define the operations and modules required for assembly.

\section{Analysis and Representation of Assembly Features Information}

According to [16] the basic core of assembly processes are "Moving Part $\mathrm{x}$ " and "Joining Parts $\mathrm{x}$ and $\mathrm{y}$ ", so that handling, mating and form features have to be used to 
derive assembly process features, transporting and joining features, definitions to determine the required transporting and joining processes and capabilities.

Handling features is a generic form of assembly information (independent on the actual position and orientation of the component within an assembly), which can be used to store and retrieve information about feeding, fixturing and grasping [9]. Handling features are generic for some assembly components, such as the base component (the component upon which all remaining assemblies are carried out). This base component is always fixtured, fed and grasped in a predefined way. For the other assembly components, mating and form features are needed as well as handling features in order to derive grasping and feeding features to determine the required transporting processes and capabilities. Figure 4 illustrates the derivation of transporting features.

Mating features are very important for representing joining relationships between assembly components/ parts, because actual joining operations occur at mating features [17]. In order for mating features to fully describe the joining operation, an expansion of these features is required; this can be achieved by combining mating features for an assembly with the geometrical entities being selected for form features. The extended mating features will be used to determine the joining features. By adding joining information (joining methods, groove shapes, joining components and entities, and joining constraints) by user, joining processes features will be derived. Figure 3 specifies different transporting (grasping, feeding, and fixturing) and joining (welding, fitting, gluing, and fastening) features, which can be derived from assembly features.

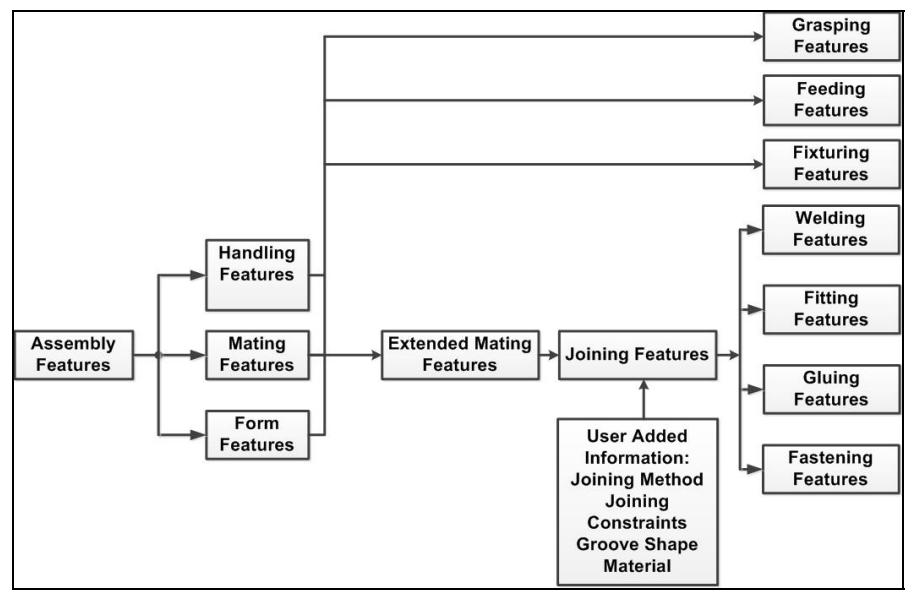

Fig. 3. Derivation of assembly processes features

The required assembly processes can be determined depending on the derived assembly process features. The required resource capabilities can be determined based on those assembly processes. Technical and functional constraints of production system's resources can be determined based on geometrical, non- geometrical, functional and constraints information of an assembly.

Derived assembly features need to be represented in a product model in order to share these features between experts in different domains in the EAS paradigm (product, process and resources). Figure 4 shows a proposed feature--based model which is based on product topology. 


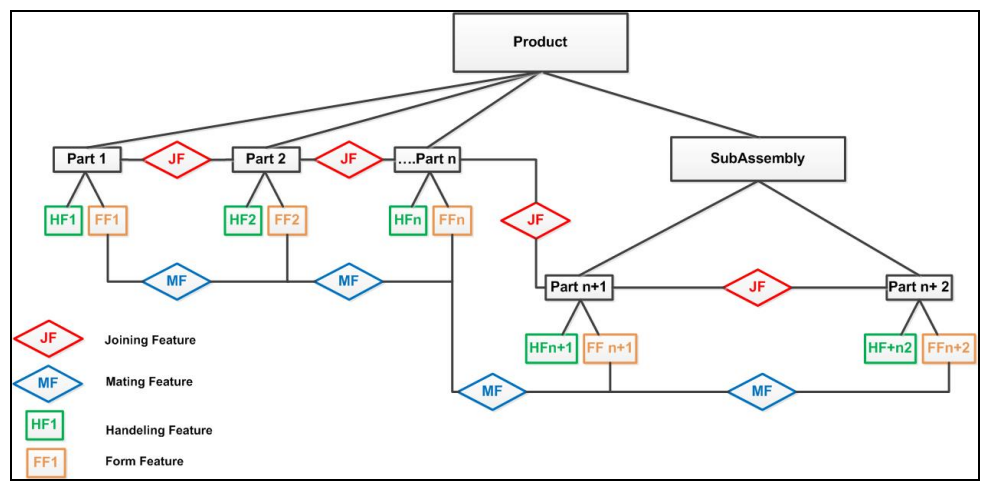

Fig. 4. Product feature-based model which is based on product topology

A product model contains a combination of different single parts. Some of these parts are assembled first to subassemblies and then to the main product. In our proposed model, handling and form features are defined for each part, while mating features are defined among form features of different parts. Joining features are defined among different parts. Figure 5 illustrates the concept of the proposed featurebased model with mapping of three parts constrained in an aligned mating relationship and screw joining relationships.

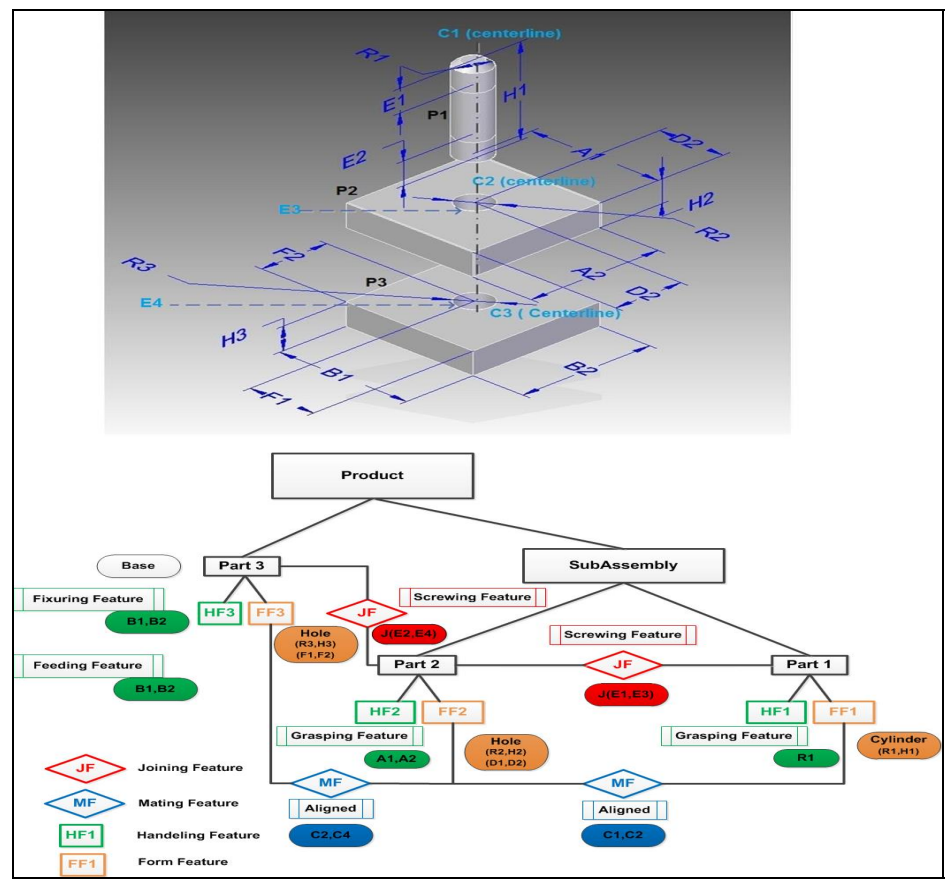

Fig. 5. Feature-based model for three parts case study example 
To share the extracted assembly information in figure 5, and to integrate product and process in to EAS, a process-feature ontology is proposed (Figure 6). According to [18] "an ontology defines the basic terms and relations comprising the vocabulary of a topic area, as well as the rules for combining terms and relations to define extensions to the vocabulary". The proposed process-feature ontology is based on an assembly design ontology (AsD) proposed by Kim [19] and the assembly process class in EAS ontology [15]. Figure 6 shows the assembly features class in this ontology, where all the joining information (constraint, tolerance, shape and configuration) is classified as instances of joining process features classes ( ex. riveting feature). By matching those instances with the skills parameters of joining modules, the required modules will be determined. The level of detail in this ontology can be extended according to the available assembly information (extracted or added by user), or according to the parameters of the joining resource capabilities in the production system.

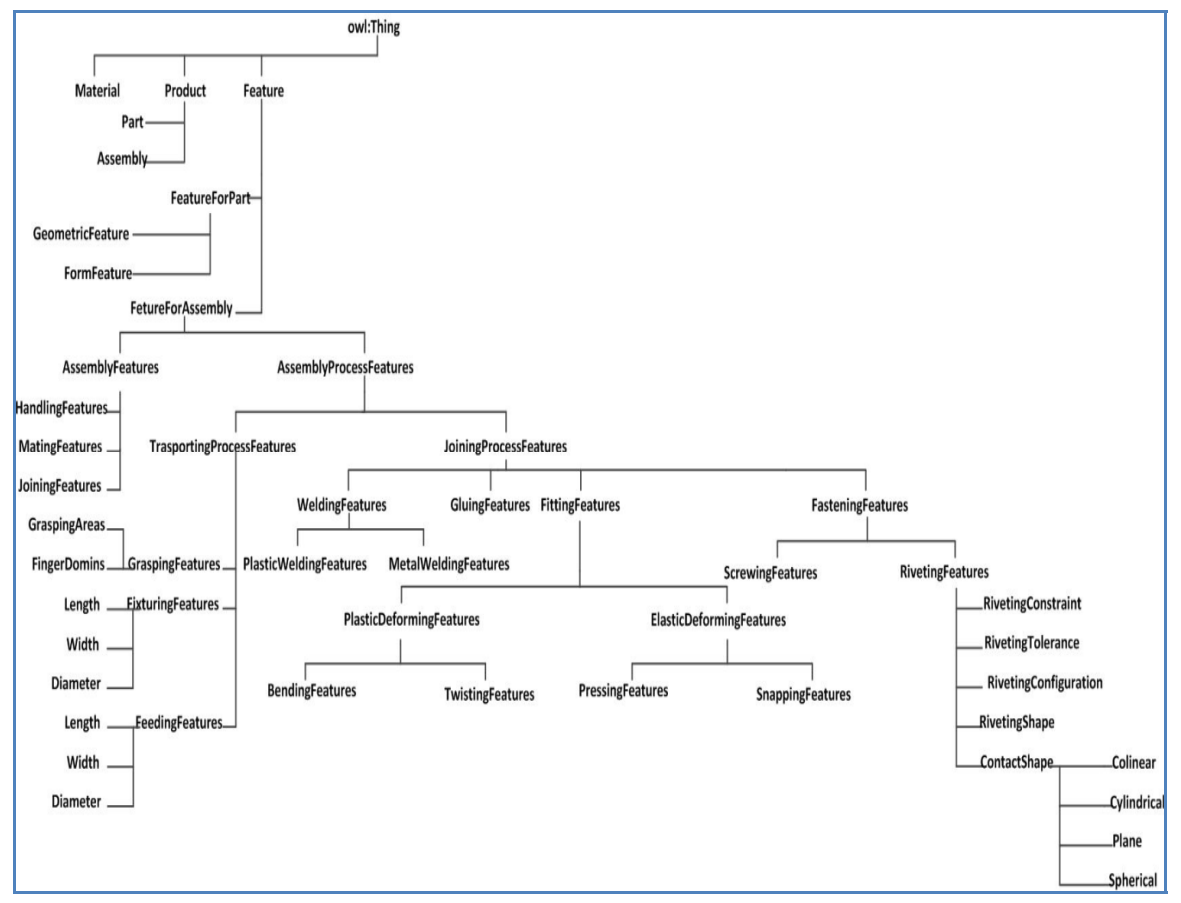

Fig. 6. Assembly features part in the process-feature ontology

\section{Conclusion}

The article presents a proposed methodology for adaptation of production systems based on matching assembly requirements, represented by proposed process features, to the processes and resources, represented by skills, in the EAS ontology. 
A proposed feature-based model is introduced based on extracted handling and form features for each part, a mating feature between parts in a product assembly. These three basic assembly features will be used to define joining and transporting features in the next stage. The complexity of the extracted features will increase gradually from the basic assembly features to the process features.

The actual extraction of assembly features from CAD files will be covered in later work. Since assembly features includes geometrical (form, handling features), nongeometrical (mating features) and functional information (constraints, configurations), a collection of extraction methods have to be used. Extraction methods for CAD information include automatic, semi-automatic and manual methods. For assembly features a semi-automatic method will be compatible with our proposed approach in this paper; since the user-added information plays an important role in defining process features.

\section{References}

1. ElMaraghy, H.A.: Flexible and reconfigurable manufacturing systems paradigms. J. International Journal of Flexible Manufacturing Systems 17(4), 261-276 (2006)

2. Koren, Y.: General RMS Characteristics, Comparison with Dedicated and Flexible Systems. In: Reconfigurable Manufacturing Systems and Transformable Factories, pp. 27-46. Springer, Dashchenko (2006)

3. Nylund, H., Salminen, K., Andersson, P.: Digital Virtual Holons - An Approach to Digital Manufacturing Systems. In: Manufacturing Systems and Technologies for the New Frontier, pp. 103-106. Springer, Mitsuishi (2008)

4. Onori, M., Semere, D.T., Lindberg, B.: Evolvable systems: An approach to self-X production. In: Huang, G.Q., Mak, K.L., Maropoulos, P.G. (eds.) DET2009 Proceedings. AISC, vol. 66, pp. 789-802. Springer, Heidelberg (2010)

5. Onori, M.: A re-engineering perspective to assembly system development. J. Industrial Robot 32(5) (2005)

6. Onori, M., Neves, P., Akillioglu, H., Maffei, A.: Dealing with the unpredictable: An Evolvable Robotic Assembly Cell. In: Int. Conf. on Changeable, Agile, Reconfigurable and Virtual Production (CARV 2011), Montreal, Canada, pp. 160-165 (2011)

7. Semere, D., Onori, M., Maffei, A., Adamietz, R.: Evolvable assembly systems: coping with variations through evolution. J. Assembly Automation 28(2), 126-133 (2008)

8. Akillioglu, H., Neves, P., Onori, M.: Evolvable assembly systems: Mechatronic Architecture Implications and Future Research. In: 3rd CIRP Conference on Assembly Technologies and Systems (2010)

9. Van Holland, W.: Assembly Features in Modelling and Planning. Ph.D. Thesis, Delft University of Technology, Holland (1997)

10. Liu, H., Nnaji, B.: Design with spatial relationships. J. Manufacture Systems 10(6) (1991)

11. Shah, J.J., Rogers, M.T.: Feature Based Modeling Shell: Design and Implementation. In: Proceedings of the ASME Conference on Computers in Engineering, Montreal, Quebec, Canada, pp. 343-354 (1988)

12. Sung, R.: Automatic Assembly Feature Recognition and Disassembly Sequence Generation. Ph.D. Thesis, Heriot-Watt University, Edinburgh, UK (2001) 
13. Pfrommer, J., Schleipen, M., Beyerer, J.: PPRS: Production skills and their relation to product, process, and resource. In: 2013 18th IEEE Conference on Emerging Technologies \& Factory Automation (ETFA), pp. 1-4 (2013)

14. Lanz, M.: Logical and Semantic Foundations of Knowledge Representation for Assembly and Manufacturing Processes. Ph.D. Thesis, Tampere Univ of Technology, Finland (2010)

15. Evolvable Ultra- Precision Assembly Systems (EUPASS): Assembling Process Ontology Specification. Technical report, EUPASS std0007 (2008)

16. Smale, D., Ratchev, S.: A Capability Model and Taxonomy for Multiple Assembly System Reconfigurations. In: 13th IFAC Symposium on Information Control Problems in Manufacturing, Moscow, pp. 1923-1928 (2009)

17. Kim, K.: Assembly operation tools for e product design and realization. Ph.D. Thesis, University of Pittsburgh, USA (2003)

18. Neches, R., Fikes, R., Finin, T., Gruber, T., Senator, T., Swartout, W.: Enabling technology for knowledge sharing. J. Al Magazine 12, 36-56 (1991)

19. Kim, K.-Y., Manley, D.G., Yang, H.: Ontology-based assembly design and information sharing for collaborative product development. J. Computer-Aided Design 38(12), $1233-1250$ (2006) 